\title{
Mycobacterium xenopi osteomyelitis of the spine: a case report
}

\author{
Fred UN Ukunda* (i) \\ Department of Orthopaedic Surgery, Chris Hani Baragwanath Academic Hospital, Johannesburg, South Africa; Department of Orthopaedic Surgery, \\ University of the Witwatersrand, Johannesburg, South Africa \\ ${ }^{*}$ Corresponding author: fredukunda@icloud.com
}

\begin{abstract}
Citation: Ukunda FUN. Mycobacterium xenopi osteomyelitis of the spine: a case report. SA Orthop J 2021;20(2):114-116. http://dx.doi. org/10.17159/2309-8309/2021/ v20n2a8
\end{abstract}

Editor: Prof. Robert Dunn, University of Cape Town, Cape Town, South Africa

Received: January 2020

Accepted: June 2020

Published: May 2021

Copyright: ( 2021 Ukunda FUN. This is an open-access article distributed under the terms of the Creative Commons Attribution Licence, which permits unrestricted use, distribution and reproduction in any medium, provided the original author and source are credited.

Funding: No funding was received for this study.

Conflict of interest: The author declares there are no conflicts of interest that are directly or indirectly related to the research.

\begin{abstract}
Background

Mycobacterium xenopi (M. xenopi) osteomyelitis is an uncommon infection which is found in immunosuppressed patients. It is reported to be a slow-growing, nonchromogenic or scotochromogenic nontuberculous mycobacterium. The lungs constitute the most common site for infection and extrapulmonary manifestations, and disseminated forms of the disease are rare. Only a few cases of spontaneous spinal involvement have been reported. We report a case of $M$. xenopi vertebral osteomyelitis of the spine.
\end{abstract}

\section{Patient and methods}

A 41-year-old female patient, HIV reactive on antiretroviral therapy with a low CD4 count of $183 \mathrm{cells} / \mathrm{mm}^{3}$, presented with clinical and radiological features in keeping with thoracic spinal tuberculosis, complicated with thoracic myelopathy. She was managed surgically with costotransversectomy and drainage of the paraspinal cold abscess. The Ziehl-Neelsen staining was negative for acid-fast bacilli. However, the histology result revealed a necrotising granulomatous inflammation. A delayed result of polymerase chain reaction (PCR)/line probe assay for Mycobacterium genus testing revealed the presence of $M$. xenopi, as the cause for the spine osteomyelitis and thoracic myelopathy. However, no $M$. xenopi susceptibility testing, and no specific photoreactivity techniques for strain identification, were performed. Anti-tuberculosis therapy (ATT) consisting of a two-month initiation phase using rifampicin, isoniazid, ethambutol and pyrazinamide, followed by a seven-month continuation phase using rifampicin and isoniazid, was initiated according to national guidelines. She was fitted with a thoraco-lumbar-sacral orthosis, and underwent a spinal rehabilitation programme. Upon receipt of the PCR result, and considering the good clinical and radiological response to ATT, a consensus was reached with the Infectious Disease Unit (IDU) to continue with ATT until 18 months due to the atypical nature of the pathogen.

\section{Results}

The patient was successfully treated with the standard TB regimen, but for a period of 18 months, and made full clinical neurological recovery, without any back pain. Furthermore, her CD4 count had also improved to 707 cells $/ \mathrm{mm}^{3}$ with a viral load reported lower than 1000 copies $/ \mathrm{ml}$.

\section{Conclusion}

This case report emphasises the importance of biopsy in suspected spinal tuberculosis and highlights the concerns with laboratory testing and the prognostic and therapeutic implications of a positive strain identification.

Level of evidence: Level 5

Keywords: Mycobacterium xenopi osteomyelitis, tuberculosis, anti-tuberculosis treatment

\section{Introduction}

Mycobacterium xenopi (M. xenopi) was first isolated in the United Kingdom in 1959 by Schwabacher from skin granulomas of a South African female toad (Xenopus laevis). It is reported to be a slow-growing, nonchromogenic or scotochromogenic nontuberculous mycobacterium (NTM) with optimal growth speed at $42{ }^{\circ} \mathrm{C} .{ }^{1}$ The lungs constitute the most common site for infection and particularly in HIV-reactive patients with CD4 counts below $100 \mathrm{cells} / \mathrm{mm}^{3}{ }^{2,3}$ Extrapulmonary manifestations and disseminated forms of the disease are rare. ${ }^{4}$ The first case of $M$. xenopi infection in humans was published in 1965 and since then only a few cases of spontaneous spinal involvement have been reported. $5-9$ However, cases of nosocomial vertebral infections have been reported following discovertebral surgery due to contaminated surgical instruments by tap water at a mean time of 5.6 years for diagnosis. ${ }^{10}$

A positive culture for $M$. xenopi raises the concern as to whether it is a contaminant or true pathogen. ${ }^{11}$ The difficulties in isolating the organism and recommendations for improving culture techniques have been reported in the literature. ${ }^{12,13}$ Increasingly, rRNA nucleic acid probe testing for $M$. xenopi is performed to confirm the diagnosis. ${ }^{14}$ The American Thoracic Society (ATS) and Infectious Diseases Society of America (IDSA) guidelines for the diagnosis 
of NTM lung disease include clinical, radiological and microbiological criteria. The microbiologic criterion requires a positive culture from at least two separate expectorated sputum samples. Unfortunately, no guidelines exist for the diagnosis of extrapulmonary manifestations. ${ }^{15,16}$ The optimal treatment for $M$. xenopi is yet to be identified. However, a recent study on mice shows significant bactericidal effect with ethambutol/ rifampicin combination with either clarithromycin or moxifloxacin. ${ }^{17}$ We report a case of thoracic spine osteomyelitis caused by $M$. xenopi, destroying three contiguous midthoracic vertebrae, causing spinal stenosis and myelopathy. The patient was successfully treated with anti-TB treatment (ATT), ${ }^{18}$ after limited cold abscess drainage. This case highlights the concerns with laboratory testing and the increased use of PCR in mycobacterial infection diagnosis.

\section{Case report}

A 41-year-old female presented with a four-month history of progressive thoracic back pain and walking difficulty. At the time of presentation, she was wheelchair-bound and she had bladder and bowel incontinence. She was HIV reactive and was on antiretroviral therapy (ART) since 2006. Her clinical evaluation revealed no obvious gibbus, but there was spastic paraplegia (American Spinal Injury Association Impairment scale [ASIA] A) ${ }^{19}$ with a T4 sensory level. Laboratory investigations revealed a raised erythrocyte sedimentation rate (ESR) of $67 \mathrm{~mm} / \mathrm{hr}$, a C-reactive protein (CRP) of $50 \mathrm{mg} / \mathrm{L}$ and a CD4 count of 187 cells $/ \mathrm{mm}^{3}$.

The thoracic spine $\mathrm{X}$-rays revealed a double heart shadow and irregularity, and collapse of the T7 and T8 vertebrae. The STIR and T2 MRI scans showed three-level thoracic spine involvement (T6-T9), with spinal stenosis and paraspinal fluid collection and increased cord signal (Figure 1).

The working diagnosis of tuberculosis (TB) of the spine was made. The differential diagnosis of an atypical mycobacterial infection, a non-mycobacterial infection (fungal) and spine neoplasm were also considered.

The patient underwent drainage of pus and caseous-like material through left-sided costo-transversectomy at T7/T8 level. Specimens were sent for Ziehl-Neelsen staining, microscopy culture and sensitivity, and histology.

The Ziehl-Neelsen staining was negative for acid-fast bacilli. However, the histology result revealed a necrotising granulomatous inflammation. No malignant cells were seen. Anti-tuberculosis therapy (ATT) consisting of a two-month initiation phase using rifampicin, isoniazid, ethambutol and pyrazinamide, followed by a seven-month continuation phase using rifampicin and isoniazid, was initiated according to national guidelines, ${ }^{18}$ and a thoracolumbar-sacral orthosis was fitted. The patient was in a wheelchair for spine rehabilitation.

At her four-month follow-up visit, the patient's back pain had reduced significantly, and her neurology had improved to ASIA C. At this stage, review of supplementary laboratory results for polymerase chain reaction (PCR)/line probe assay for Mycobacterium genus testing revealed the presence of $M$. xenopi.
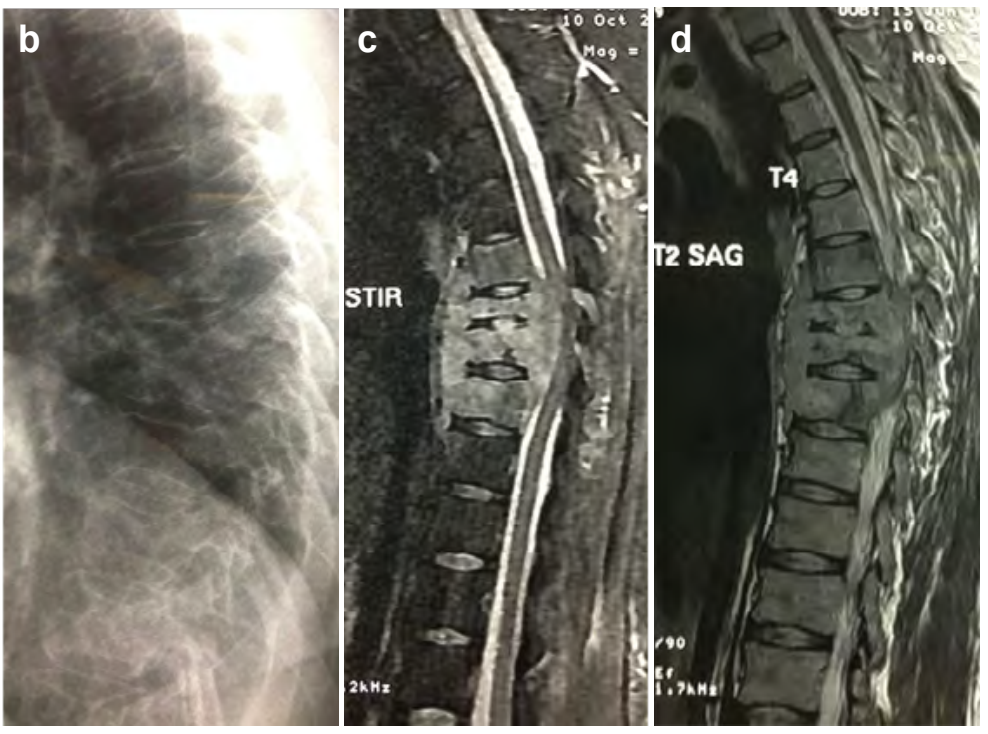

Figure 1. The thoracic spine X-rays showed in (a) double heart shadow and (b) irregularity and the collapse araspinal fluid collection, as well as cord signal.

However, no $M$. xenopi susceptibility testing, and no specific photoreactivity techniques for strain identification were performed. The culture result showed no growth. Her CD4 count had also improved to 707 cells $/ \mathrm{mm}^{3}$ with a viral load reported lower than 1000 copies/ml. The thoracic spine X-rays (Figure 2) showed no progression of vertebral destruction with reduction of the paraspinal shadow seen at presentation. Considering the good clinical and radiological response to ATT, a consensus was reached with the Infectious Disease Unit (IDU) to continue with ATT until 18 months due to the atypical nature of the pathogen.

At the next follow-up visit, the patient's neurology had improved to ASIA D, she was mobilising with crutches and she had good recovery of bowel and bladder control. The ATT was continued until she completed 18 months. At her last follow-up visit, she had full neurological recovery without any back pain and she returned to work.

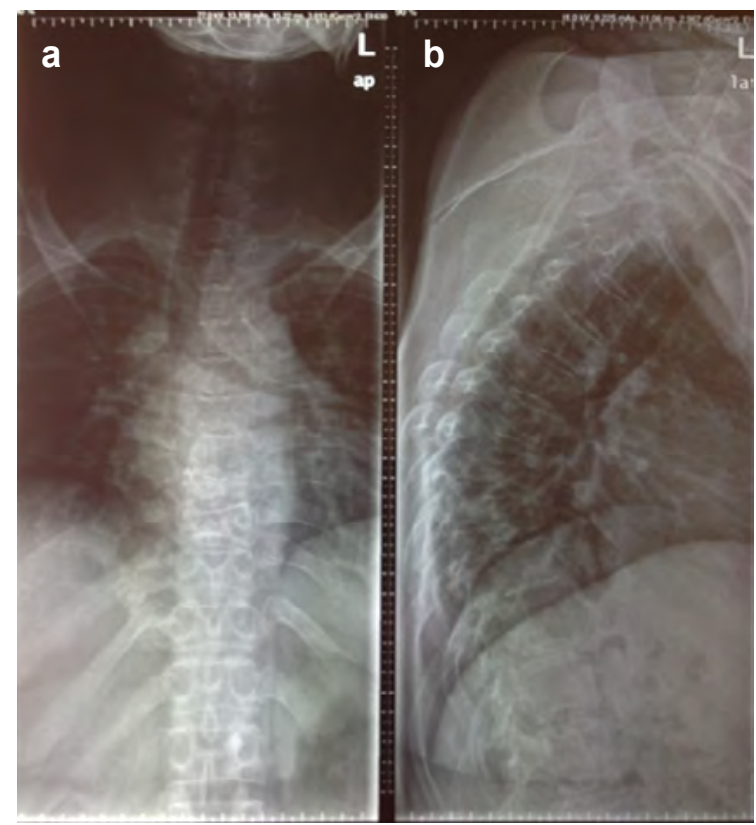

Figure 2. Last follow-up thoracic spine X-rays show no progression of vertebral destruction with reduction of the paraspinal shadow. 


\section{Discussion}

The majority of clinical cases of $M$. xenopi have been found in the pulmonary specimens of HIV-reactive patients. Although extremely rare, Kulasegaram et al. ${ }^{16}$ report that extrapulmonary spinal M. xenopi has a clinical presentation somewhat similar to M. tuberculosis, but the progression seems slower. In most case reports, treatment was conservative with variable results. ${ }^{2,4}$ Our patient confirms these observations, as she had a presentation like that of spinal $M$. tuberculosis, was HIV reactive with low immunity (CD4 count) and she responded well to ATT after limited cold abscess drainage.

Tissue diagnosis in the form of CT-guided, percutaneous or open vertebral biopsy, is always required. ${ }^{16}$ Photoreactivity testing to reveal a non-chromogen isolate and, increasingly, confirmation by rRNA nucleic acid probe testing are performed. ${ }^{1,15}$ In this case, an open biopsy was undertaken. The microbiological diagnosis of M. xenopi was made from the laboratory PCR supplementary testing of the drainage specimen. In the absence of strain identification, the need for repeat biopsy was considered. However, concerns regarding the assurance of a positive yield, the invasive nature of the procedure and the good clinical and radiological response to ATT led us to regard the procedure as unjustified and it was not performed. Currently, although the ATS diagnostic criteria are designed for lung disease, many authors still recommend their awareness as a good tool for determining clinical relevance. ${ }^{14,15}$

According to the British Thoracic Society, the treatment in pulmonary disease is reported to be difficult with high failure, relapse and mortality rates, despite prolonged courses of rifampicin, ethambutol and isoniazid. ${ }^{20}$ The ATS/IDSA (American Thoracic Society/Infectious Diseases Society of America) recommends a combination regimen that includes either streptomycin or clarithromycin for a duration of 18 to 24 months. Ongoing trials with macrolides and quinolones are yet to produce results. Other reports suggest there is no correlation between in vitro susceptibility and clinical response.

This patient was successfully treated with standard TB regimen, but for a period of 18 months as recommended by the IDU, and she made full clinical (neurological) recovery. The favourable response to the standard TB regimen suggests that $M$. xenopi was susceptible to at least one of the components of the regimen. This emphasises the unresolved debate on the exact susceptibility of $M$. xenopi and its value in clinical practice. Therefore, greater awareness of the ATS nontuberculous mycobacteria management guidelines is recommended in the literature, to optimise the clinical response. ${ }^{17}$

\section{Conclusion}

In the presense of HIV co-infection, atypical mycobacterial infection must be considered in the differential diagnosis. This case report emphasises the importance of biopsy in suspected spinal TB cases, not only to establish the diagnosis but also for strain identification and prognostic and therapeutic implications.

\section{Acknowledgements}

We would like to acknowledge the support of the Orthopaedic Spine Team at Chris Hani Baragwanath Academic Hospital, South Africa, and the contributions of Dr A Mjuza.

\section{Ethics statement}

The study was approved by the Human Research Ethics Committee at the University of the Witwatersrand (M190943), and the Chris Hani Baragwanath Hospital Management granted permission for it to be undertaken.

All procedures were in accordance with the ethical standards of the responsible committee on human experimentation (institutional and national) and with the Helsinki Declaration of 1975 , as revised in 2008.
Declaration

The author declares authorship of this article and that they have followed sound scientific research practice. This research is original and does not transgress plagiarism policies.

\section{Author contributions}

FU formulated the research aims and objectives, collected the data and prepared the manuscript.

\section{ORCID}

Ukunda FUN https://orcid.org/0000-0002-9284-6358

\section{References}

1. Schwabacher H. A strain of Mycobacterium isolated from skin lesions of a cold-blooded animal, Xenopus laevis, and its relation to atypical acid-fast bacilli occurring in man. Epidemiology \& Infection. 1959;57(1):57-67.

2. Danesh-Clough T, Theis J-C, Van der Linden A. Mycobacterium xenopi infection of the spine: a case report and literature review. Spine. 2000;25(5):626.

3. El-Helou P, Rachlis A, Fong I, et al. Mycobacterium xenopi infection in patients with human immunodeficiency virus infection. Clinical Infectious Diseases. 1997;25(2):206-10.

4. Donnabella V, Salazar-Schicchi J, Bonk S, et al. Increasing incidence of Mycobacterium xenopi at Bellevue hospital: an emerging pathogen or a product of improved laboratory methods? Chest. 2000;118(5):1365-70.

5. Jiva TM, Jacoby HM, Weymouth LA, et al. Mycobacterium xenopi: innocent bystander or emerging pathogen? Clinical Infectious Diseases. 1997;24(2):225-32.

6. Jones PG, Schrager MA, Zabransky RJ. Pott's disease caused by Mycobacterium xenopi. Clinical Infectious Diseases. 1995;21(5):1352.

7. Kerbiriou L, Ustianowski A, Johnson M, et al. Human immunodeficiency virus type 1-related pulmonary Mycobacterium xenopi infection: a need to treat? Clinical Infectious Diseases. 2003;37(9):1250-54.

8. Manfredi R, Nanetti A, Tadolini M, et al. Role of Mycobacterium xenopi disease in patients with HIV infection at the time of highly active antiretroviral therapy (HAART). Comparison with the pre-Haart period. Tuberculosis. 2003;83(5):319-28.

9. Ormerod P. A step forward in the evidence-based treatment of opportunist mycobacteria. BMJ Publishing Group Ltd; 2001.

10. Astagneau P, Desplaces N, Vincent V, et al. Mycobacterium xenopi spinal infections after discovertebral surgery: investigation and screening of a large outbreak. Lancet. 2001;358(9283):747-51.

11. Prosser AJ. Spinal infection with Mycobacterium xenopi. Tubercle. 1986;67(3):229-32.

12. Andréjak C, Almeida DV, Tyagi S, et al. Improving existing tools for Mycobacterium xenopi treatment: assessment of drug combinations and characterization of mouse models of infection and chemotherapy. J Antimicrob Chemother. 2013;68(3):659-65.

13. Rahman M, Phongsathorn V, Hughes $T$, et al. Spinal infection by Mycobacterium xenopi in a non-immunosuppressed patient. Tuber Lung Dis. 1992;73(6):392-95.

14. Meybeck A, Fortin C, Abgrall S, et al. Spondylitis due to Mycobacterium xenopi in a human immunodeficiency virus type 1-infected patient: case report and review of the literature. J Clin Microbiol. 2005;43(3):1465-66.

15. Van Ingen J, Boeree MJ, De Lange WC, et al. Mycobacterium xenopi clinical relevance and determinants, the Netherlands. Emerg Infect Dis. 2008;14(3):385.

16. Kulasegaram R, Richardson D, Macrae B, De Ruiter A. Mycobacterium xenop osteomyelitis in a patient on highly active antiretroviral therapy (HAART). Int J STD AIDS. 2001;12(6):404-406.

17. Alfreijat M, Ononiwu C, Sexton C. Pott's disease: A case of Mycobacterium xenopi infection of the spine. J Community Hosp Intern Med Perspect. 2013;2(4):20150.

18. Department of Health. National Tuberculosis Management Guidelines. Pretoria: Department of Health; 2014.

19. Roberts TT, Leonard GR, Cepela DJ. Classifications in brief: American Spinal Injury Association (ASIA) Impairment Scale. Clin Orthop Relat Res. 2017;475(5):1499-504

20. Research Committee of the British Thoracic Society. First randomised trial of treatments for pulmonary disease caused by $M$ avium intracellulare, $M$ malmoense, and $M$ xenopi in HIV negative patients: rifampicin, ethambutol and isoniazid versus rifampicin and ethambutol. Thorax. 2001;56(3):167-72. 